\title{
Novel Device to Use during Face Mask Ventilation in Edentulous Patients
}

\author{
Monish S. Raut* \\ Department of Cardiac Anesthesia, Sir Ganga Ram Hospital, Old Rajinder Nagar, New Delhi Pin 110060, India
}

\begin{abstract}
Mask ventilation is essential in the event of difficult intubation. Incidence of difficult mask ventilation is reported to be $1.5-7.8 \%$ in the general population and $16 \%$ in edentulous patients. ${ }^{1-4}$ Hence, difficult mask ventilation is of paramount importance for safe airway management. In edentulous patients, ineffective seating of the standard mask to the face causes decreased contact between the mask and the cheeks, resulting in leakage of air during positive pressure ventilation..$^{5}$ Dentures help in maintaining better face mask fit; however, this imposes the risk of aspiration and swallowing during induction of anesthesia. ${ }^{2,4}$ The safer practice, therefore, involves removal of denture before the anesthesia administration.

Placing the caudal end of the mask between the inferior lip and the alveolar ridge, and holding the lower lip face mask placement with a two-handed grip have been suggested for adequate mask ventilation in such edentulous patients. ${ }^{4}$ But, such techniques to support the face mask are physically cumbersome and can, themselves, cause air leak during bag and mask ventilation. Nasal positive-pressure ventilation by face mask has also been reported to be an effective mode of ventilation in edentulous patients. ${ }^{6}$ Placing the nasal mask over the upper lip gives good contact of the mask with tissues that have maxillary support. Moreover, nasal mask ventilation has been reported to give better air delivery and to have a reduced risk of leaks; however, this method can cause oral air leakage. ${ }^{7}$
\end{abstract}

We suggest use of a new mask support device consisting of a soft cotton cloth pad of square shape with breadth of 2 inches and a circular aperture in the center (Fig. 1). This cotton pad is placed so as to cover the patient's mouth and nostrils, with the central hole over the nostrils. While ventilating the edentulous patient, a standard face mask is placed over this cotton pad positioned in above-mentioned way (Fig. 2). Ventilation through the standard face mask can then be easily achieved through the central open aperture of the pad. Leakage around the mask can be prevented by an interposed pad placed between the mask edges and the facial contour.

The advantage of this preliminary support device is the simplicity of its design and the fact that it can be easily produced using readily available materials (i.e. a cotton pad). It offers ease and comfort for holding the face mask in place, ultimately providing better ventilation to the patient without any air leakage. It can

Received: October 06, 2016; Revised: February 23, 2017; Accepted: February 25, 2017

${ }^{*}$ Correspondence to: Monish Raut, Department of Cardiac Anesthesia, Sir Ganga Ram Hospital, Old Rajinder Nagar, New Delhi Pin 110060, India. Tel: +918377836463, E-mail: drmonishraut@gmail.com

How to cite this article: Raut MS. Novel Device to Use during Face Mask Ventilation in Edentulous Patients. Explor Res Hypothesis Med 2017;2(1):28-29. doi: 10.14218/ERHM.2016.00015. be used for all sizes of face mask. Hence, the only drawback it may carry is the possibility of contamination and wetting due to inadvertent oral secretions. Generating a similar device from soft gelbase/latex can solve this problem. Future research studies are warranted to establish its practical applicability in clinical practice.

\section{Conflict of interest}

The author has no confict of interest related to this publication.

Author contributions

Manuscript writing (MSR).

\section{References}

[1] Yildiz TS, Solak M, Toker K. The incidence and risk factors of difficult mask ventilation. J Anesth 2005;19(1):7-11. doi:10.1007/s00540004-0275-z.

[2] Langeron O, Masso E, Huraux C, Guggiari M, Bianchi A, Coriat $\mathrm{P}$, et al. Prediction of difficult mask ventilation. Anesthesiology 2000;92(5):1229-1236

[3] Kheterpal S, Han R, Tremper KK, Shanks A, Tait AR, O'Reilly M, et al. Incidence and predictors of difficult and impossible mask ventilation. Anesthesiology 2006;105(5):885-891.

[4] Racine SX, Solis A, Hamou NA, Letoumelin P, Hepner DL, Beloucif $S$, et al. Face mask ventilation in edentulous patients: a comparison of mandibular groove and lower lip placement. Anesthesiology 2010;112(5):1190-1193. doi:10.1097/ALN.0b013e3181d5dfea.

[5] Kheterpal S, Martin L, Shanks AM, Tremper KK. Prediction and outcomes of impossible mask ventilation: a review of 50,000 anesthetics. Anesthesiology 2009;110(4):891-897. doi:10.1097/ ALN.0b013e31819b5b87.

[6] Liang Y, Kimball WR, Kacmarek RM, Zapol WM, Jiang Y. Nasal ventilation is more effective than combined oralnasal ventilation during induction of general anesthesia in adult subjects. Anesthesiology 108(6):998-1003. doi:10.1097/ALN.0b013e318174f027.

[7] Kapoor MC, Rana S, Singh AK, Vishal V, Sikdar I. Nasal mask ventilation is better than face mask ventilation in edentulous patients. J Anaesthesiol Clin Pharmacol 2016;32(3):314-318. doi:10.4103/09709185.168262. 
Raut MS.: Novel face mask support device

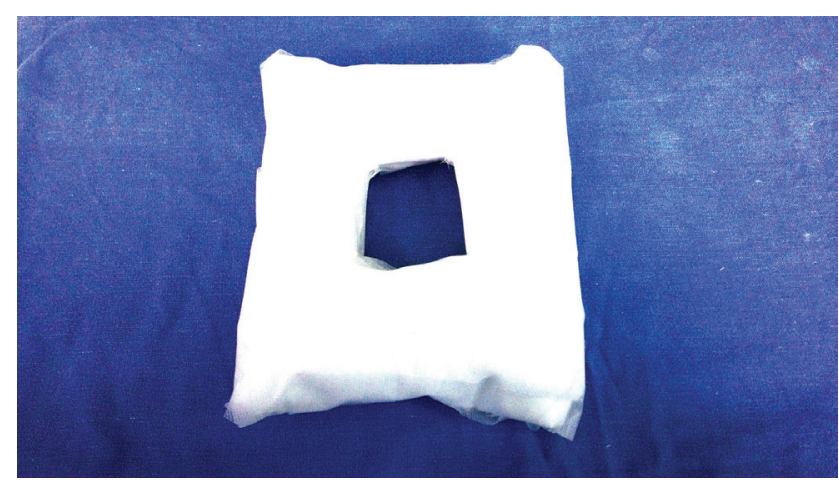

Fig. 1. Raut's face mask support device with central aperture.
Explor Res Hypothesis Med

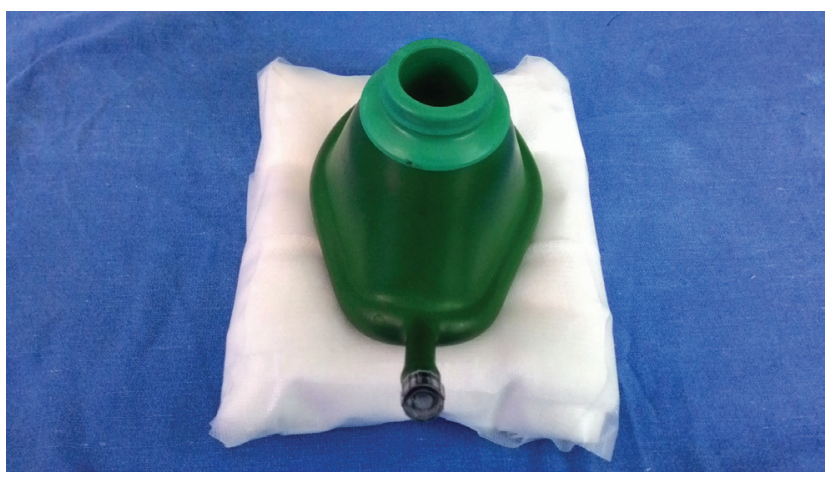

Fig. 2. Standard face mask placed over the central aperture of Raut's face mask support device. 